\title{
DERIVATIVE-FREE CHARACTERIZATIONS OF COMPACT GENERALIZED COMPOSITION OPERATORS BETWEEN ZYGMUND TYPE SPACES
}

\author{
MIKAEL LINDSTRÖM ${ }^{\bowtie}$ and AMIR H. SANATPOUR
}

(Received 17 March 2009)

\begin{abstract}
We give derivative-free characterizations for bounded and compact generalized composition operators between (little) Zygmund type spaces. To obtain these results, we extend Pavlović's corresponding result for bounded composition operators between analytic Lipschitz spaces.
\end{abstract}

2000 Mathematics subject classification: primary 47B38; secondary 47B33, 30D55, 46E15.

Keywords and phrases: Zygmund spaces, Lipschitz spaces, weighted composition operators, generalized composition operators.

\section{Introduction and main results}

Let $H(D)$ be the space of all analytic functions on the open unit disc $D$. Then, for $0<\alpha<\infty$, we denote by $\mathcal{B}^{\alpha}$ the Bloch type space of all functions $f \in H(D)$ satisfying

$$
\sup _{z \in D}(1-|z|)^{\alpha}\left|f^{\prime}(z)\right|<\infty
$$

The space $\mathcal{B}^{\alpha}$ is a Banach space with the norm

$$
\|f\|_{\mathcal{B}^{\alpha}}=|f(0)|+\sup _{z \in D}(1-|z|)^{\alpha}\left|f^{\prime}(z)\right| .
$$

The little Bloch type space, denoted by $\mathcal{B}_{0}^{\alpha}$, consists of those functions $f \in \mathcal{B}^{\alpha}$ such that

$$
\lim _{|z| \rightarrow 1}(1-|z|)^{\alpha}\left|f^{\prime}(z)\right|=0 .
$$

For each $0<\alpha<1$ the space $\mathcal{B}^{1-\alpha}$ can be identified with the analytic Lipschitz space $H \Lambda_{\alpha}:=H(D) \cap \Lambda_{\alpha}(D)$, where $\Lambda_{\alpha}(D)$ is the Lipschitz space of order $\alpha$ of

(C) 2010 Australian Mathematical Publishing Association Inc. 0004-9727/2010 \$16.00 
all functions $f \in C(D)$ with

$$
p_{\alpha}(f)=\sup _{\substack{z, w \in D \\ z \neq w}} \frac{|f(z)-f(w)|}{|z-w|^{\alpha}}<\infty .
$$

The space $\Lambda_{\alpha}(D), 0<\alpha \leq 1$, becomes a Banach space when equipped with the norm

$$
\|f\|_{\Lambda_{\alpha}}=\|f\|_{D}+p_{\alpha}(f),
$$

and $H \Lambda_{\alpha} \subset A(D):=H(D) \cap C(\bar{D})$ is a closed subspace of $\Lambda_{\alpha}(D)$. For $0<\alpha<1$ the little Lipschitz space of order $\alpha$, denoted by $\Lambda_{\alpha}^{0}(D)$, is the space of those functions $f \in \Lambda_{\alpha}(D)$ such that

$$
\lim _{\substack{|z-w| \rightarrow 0 \\ z, w \in D}} \frac{|f(z)-f(w)|}{|z-w|^{\alpha}}=0 .
$$

The space $\Lambda_{\alpha}^{0}(D)$ is a closed subspace of $\Lambda_{\alpha}(D)$ and also $H \Lambda_{\alpha}^{0}:=H(D) \cap \Lambda_{\alpha}^{0}(D)$ is a closed subspace of $H \Lambda_{\alpha}$.

Let $\psi \in H(D)$ and $\varphi$ be an analytic self-map of $D$. The weighted composition operator $\psi C_{\varphi}$ is the operator given by $\left(\psi C_{\varphi} f\right)(z)=\psi(z) f(\varphi(z))$. In the special case of $\psi=1$ we get the composition operator $\left(C_{\varphi} f\right)(z)=f(\varphi(z))$. Boundedness and compactness of composition operators on Bloch spaces were first studied by Roan [15] and later by Madigan [8] and Madigan and Matheson [9]. Moreover, Ohno et al. studied weighted composition operators between Bloch type spaces in [12].

Using a result of Madigan [8], Pavlović gave derivative-free characterizations of bounded composition operators between analytic Lipschitz spaces $H \Lambda_{\alpha}$ in [14]. His proof is also based on a consequence of the Schwarz lemma which he had used earlier in [13] to give a simple proof of Dyakonov's characterization of analytic weighted Lipschitz functions in terms of their moduli [5]. Indeed, he proved that for each $f \in$ $H(D)$ and each $z \in D$, there exists $w_{z} \in D$ such that $\left|w_{z}-z\right| \leq \frac{1}{2}(1-|z|)<1-|z|$ and

$$
\left|f^{\prime}(z)\right| \leq 4 \frac{\left|f\left(w_{z}\right)\right|-|f(z)|}{1-|z|} .
$$

In this paper, by using the above-mentioned consequence of the Schwarz lemma, we give derivative-free characterizations of boundedness and compactness for the recently introduced concept of generalized composition operators on (little) Zygmund type spaces.

For $g \in H(D)$ and $\varphi$ an analytic self-map of $D$, Li and Stević in [6] introduced the generalized composition operator $C_{\varphi}^{g}$ given by

$$
\left(C_{\varphi}^{g} f\right)(z)=\int_{0}^{z} f^{\prime}(\varphi(\xi)) g(\xi) d \xi
$$

If $g=\varphi^{\prime}$ then $C_{\varphi}^{\varphi^{\prime}}$ is the composition operator $C_{\varphi}$ up to a constant. $\mathrm{Li}$ and Stević investigated boundedness and compactness of generalized composition operators 
between (little) Bloch type spaces and (little) Zygmund spaces, where the Zygmund space $\mathcal{Z}$ is the class of all functions $f \in H(D) \cap C(\bar{D})$ with

$$
\sup _{\substack{i \theta \in \partial D \\ h>0}} \frac{\left|f\left(e^{i(\theta+h)}\right)+f\left(e^{i(\theta-h)}\right)-2 f\left(e^{i \theta}\right)\right|}{h}<\infty .
$$

Boundedness of composition operators on $\mathcal{Z}$ was first studied by Choe et al. in [3].

By [4, Theorem 5.3], an analytic function $f$ belongs to $\mathcal{Z}$ if and only if $f^{\prime} \in \mathcal{B}^{1}$, or equivalently $\sup _{z \in D}(1-|z|)\left|f^{\prime \prime}(z)\right|<\infty$. For $0<\alpha<\infty$ we denote by $\mathcal{Z}^{\alpha}$ the Zygmund type space of those functions $f \in H(D)$ satisfying

$$
\sup _{z \in D}(1-|z|)^{\alpha}\left|f^{\prime \prime}(z)\right|<\infty
$$

The space $\mathcal{Z}^{\alpha}$ is a Banach space with the norm

$$
\|f\|_{\mathcal{Z}^{\alpha}}=|f(0)|+\left|f^{\prime}(0)\right|+\sup _{z \in D}(1-|z|)^{\alpha}\left|f^{\prime \prime}(z)\right| .
$$

The little Zygmund type space, denoted by $\mathcal{Z}_{0}^{\alpha}$, is the closed subspace of $\mathcal{Z}^{\alpha}$ consisting of those functions $f \in \mathcal{Z}^{\alpha}$ with

$$
\lim _{|z| \rightarrow 1}(1-|z|)^{\alpha}\left|f^{\prime \prime}(z)\right|=0 .
$$

Our main results are the following derivative-free characterizations of boundedness and compactness of $C_{\varphi}^{g}$ between (little) Zygmund type spaces. We will prove these theorems after first extending Pavlović's results to compact weighted composition operators between (little) analytic Lipschitz spaces. Indeed, we reduce the study of generalized composition operators on Zygmund type spaces to weighted composition operators on Bloch type spaces. This also leads to very simple proofs of Theorems 5 and 6 in [6].

THEOREM 1.1. Let $0<\alpha, \beta<1$ and $g, \varphi \in H(D)$ where $\varphi$ is a self-map of $D$.

(i) $\quad C_{\varphi}^{g}: \mathcal{Z}^{\alpha} \rightarrow \mathcal{Z}^{\beta}$ is bounded if and only if $g \in \mathcal{B}^{\beta}$ and $|g|(1-|\varphi|)^{1-\alpha} \in$ $\Lambda_{1-\beta}(D)$.

(ii) If $g, \varphi \in \mathcal{B}_{0}^{\beta}$, then $C_{\varphi}^{g}: \mathcal{Z}^{\alpha} \rightarrow \mathcal{Z}^{\beta}$ is compact if and only if $|g|(1-|\varphi|)^{1-\alpha} \in$ $\Lambda_{1-\beta}^{0}(D)$.

THEOREM 1.2. Let $0<\alpha, \beta<1$ and $g, \varphi \in H(D)$ where $\varphi$ is a self-map of $D$. Then $C_{\varphi}^{g}: \mathcal{Z}_{0}^{\alpha} \rightarrow \mathcal{Z}_{0}^{\beta}$ is bounded if and only if the following hold:

(1) $g \in \mathcal{B}_{0}^{\beta}$;

(2) $|g|(1-|\varphi|)^{1-\alpha} \in \Lambda_{1-\beta}(D)$;

(3) $g \varphi \in \mathcal{B}_{0}^{\beta}$.

THEOREM 1.3. Let $0<\alpha, \beta<1$ and $g, \varphi \in H(D)$ where $\varphi$ is a self-map of $D$. Then the following statements are equivalent: 
(i) $C_{\varphi}^{g}: \mathcal{Z}_{0}^{\alpha} \rightarrow \mathcal{Z}_{0}^{\beta}$ is compact;

(ii) $\lim _{|z| \rightarrow 1}|g(z)|\left((1-|z|)^{\beta} /(1-|\varphi(z)|)^{\alpha}\right)\left|\varphi^{\prime}(z)\right|=0$ and $g \in \mathcal{B}_{0}^{\beta}$;

(iii) $|g|(1-|\varphi|)^{1-\alpha} \in \Lambda_{1-\beta}^{0}(D)$ and $g \in \mathcal{B}_{0}^{\beta}$.

\section{Derivative-free characterizations}

By the definition of little Lipschitz spaces (1.1), one can see that $H \Lambda_{\alpha}^{0}$ is the closure of polynomials in $D$ and therefore by [17, Theorem 7.10] we have the following lemma.

LEMMA 2.1. Let $f \in H(D)$ and $0<\alpha<1$. Then $f \in H \Lambda_{\alpha}^{0}$ if and only if

$$
\lim _{|z| \rightarrow 1}(1-|z|)^{1-\alpha}\left|f^{\prime}(z)\right|=0 .
$$

The next lemma extends this result to $C^{1}$-functions.

LEMMA 2.2. Let $u$ be a real valued $C^{1}$-function on $D$ and $0<\alpha<1$. If

$$
\lim _{|z| \rightarrow 1}(1-|z|)^{1-\alpha}|\nabla u(z)|=0,
$$

then $u \in \Lambda_{\alpha}^{0}(D)$.

PROOF. Let $\varepsilon>0$ and choose $\delta_{1}>0$ such that

$$
|\nabla u(z)|<\frac{\varepsilon}{(1-|z|)^{1-\alpha}}
$$

for each $z \in D$ with $1-\delta_{1} \leq|z|<1$. Since $u$ is a $C^{1}$-function, by using Lagrange's theorem one can see that $u \in \Lambda_{\alpha}^{0}\left(\left(1-\frac{1}{3} \delta_{1}\right) D\right)$ and therefore there exists $\delta_{2}>0$ such that

$$
\frac{|u(z)-u(w)|}{|z-w|^{\alpha}}<\varepsilon,
$$

for every $z, w \in\left(1-\frac{1}{3} \delta_{1}\right) D$ with $0<|z-w|<\delta_{2}$. Choose $0<\delta_{3}<1$ so that for each $z, w \in D \backslash\left(1-\frac{2}{3} \delta_{1}\right) D$ with $|z-w|<\delta_{3}$ the line segment between $z$ and $w$ lies in $D \backslash\left(1-\delta_{1}\right) D$. Let $\delta=\min \left\{\frac{1}{3} \delta_{1}, \delta_{2}, \delta_{3}\right\}$ and $z, w \in D$ with $|z-w|<\delta$. If $z$ or $w$ belongs to $\left(1-\frac{2}{3} \delta_{1}\right) D$ then $z, w \in\left(1-\frac{1}{3} \delta_{1}\right) D$ and $|u(z)-u(w)| /|z-w|^{\alpha}<\varepsilon$ by (2.2). If $z, w \in D \backslash\left(1-\frac{2}{3} \delta_{1}\right) D$ then by using (2.1) and the same lines of argument as in [16, Lemma 6.4.8] one can find a constant $C$, independent of $z$ and $w$, such that $|u(z)-u(w)|<C \varepsilon|z-w|^{\alpha}$. This completes the proof.

It is worth mentioning that by using (1.2) and Lemma 2.1 we have the following corollary, extending the result of [14, Theorem B] for little analytic Lipschitz spaces (see also [5, 13]).

Corollary 2.3. Let $u \in H(D)$ and $0<\alpha<1$. Then $u \in \Lambda_{\alpha}^{0}(D)$ if and only if $|u| \in \Lambda_{\alpha}^{0}(D)$. 
Proof. Clearly if $u \in \Lambda_{\alpha}^{0}(D)$, then $|u| \in \Lambda_{\alpha}^{0}(D)$. Now let $|u| \in \Lambda_{\alpha}^{0}(D)$. By (1.2) for each $z \in D$ there exists $w_{z} \in D$ such that $\left|w_{z}-z\right|<1-|z|$ and

$$
\left|u^{\prime}(z)\right| \leq 4 \frac{\left|u\left(w_{z}\right)\right|-|u(z)|}{1-|z|} .
$$

Therefore,

$$
\frac{1}{4}\left|u^{\prime}(z)\right|(1-|z|)^{1-\alpha} \leq \frac{\left|u\left(w_{z}\right)\right|-|u(z)|}{(1-|z|)^{\alpha}} \leq \frac{|| u\left(w_{z}\right)|-| u(z)||}{\left|w_{z}-z\right|^{\alpha}},
$$

which implies that $\lim _{|z| \rightarrow 1}\left|u^{\prime}(z)\right|(1-|z|)^{1-\alpha}=0$, since $\left|w_{z}-z\right|<1-|z|$ and

$$
\frac{|| u(\zeta)|-| u(\eta)||}{|\zeta-\eta|^{\alpha}} \rightarrow 0 \quad \text { as }|\zeta-\eta| \rightarrow 0
$$

Therefore $u \in \Lambda_{\alpha}^{0}(D)$ by Lemma 2.1.

Let $\gamma>0$ and consider the following linear maps between Zygmund type spaces and Bloch type spaces of order $\gamma$ :

$$
\begin{gathered}
S: \mathcal{Z}^{\gamma} \rightarrow \mathcal{B}^{\gamma}, \quad S h=h^{\prime} \\
T: \mathcal{B}^{\gamma} \rightarrow \mathcal{Z}^{\gamma}, \quad(T h)(z)=\int_{0}^{z} h(\xi) d \xi .
\end{gathered}
$$

The operators $S$ and $T$ are bounded, indeed $\|S h\|_{\mathcal{B}^{\gamma}} \leq\|h\|_{\mathcal{Z}^{\gamma}}$ and $\|T h\|_{\mathcal{Z}^{\gamma}}=\|h\|_{\mathcal{B}^{\gamma}}$.

Let $\varphi$ be an analytic self-map of $D$ and $g \in H(D)$. For the positive numbers $\alpha, \beta$ consider the generalized composition operator $C_{\varphi}^{g}: \mathcal{Z}^{\alpha} \rightarrow \mathcal{Z}^{\beta}$ and the following diagram:

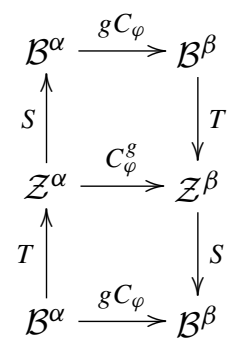

Applying (2.3) and (2.4) gives

$$
\begin{aligned}
& T \circ g C_{\varphi} \circ S=C_{\varphi}^{g}, \\
& S \circ C_{\varphi}^{g} \circ T=g C_{\varphi},
\end{aligned}
$$

which yield the following result.

Proposition 2.4. Let $\alpha, \beta>0$ and $g, \varphi \in H(D)$ where $\varphi$ is a self-map of $D$. Then $C_{\varphi}^{g}: \mathcal{Z}^{\alpha} \rightarrow \mathcal{Z}^{\beta}$ is bounded (compact) if and only if $g C_{\varphi}: \mathcal{B}^{\alpha} \rightarrow \mathcal{B}^{\beta}$ is bounded (compact). 
Note that [6, Theorems 5 and 6] follow from Proposition 2.4 in the special case of $\alpha=\beta=1$ along with [12, Theorems 2.1(ii) and 3.1(ii)].

Since $S\left(\mathcal{Z}_{0}^{\gamma}\right) \subseteq \mathcal{B}_{0}^{\gamma}$ and $T\left(\mathcal{B}_{0}^{\gamma}\right) \subseteq \mathcal{Z}_{0}^{\gamma}$, one can also consider the commutativity of diagram (2.5) for little Zygmund type spaces and little Bloch type spaces. Hence, we get the following proposition.

Proposition 2.5. Let $\alpha, \beta>0$ and $g, \varphi \in H(D)$ where $\varphi$ is a self-map of $D$. Then $C_{\varphi}^{g}: \mathcal{Z}_{0}^{\alpha} \rightarrow \mathcal{Z}_{0}^{\beta}$ is bounded (compact) if and only if $g C_{\varphi}: \mathcal{B}_{0}^{\alpha} \rightarrow \mathcal{B}_{0}^{\beta}$ is bounded (compact).

Therefore in order to prove Theorems 1.1, 1.2, and 1.3 we need to solve the derivative-free problem for weighted composition operators between (little) Bloch type spaces or (little) analytic Lipschitz spaces.

Pavlović used [8, Theorem 4] to give a derivative-free characterization for the boundedness of a composition operator $C_{\varphi}: H \Lambda_{\alpha} \rightarrow H \Lambda_{\beta}$ in [14, Theorem A]. By the same method of proof as in [14, Theorem A] and using [12, Theorem 2.1(i)] one also has the following version of [14, Theorem A] for the weighted composition operators.

THEOREM 2.6. Let $0<\alpha, \beta \leq 1$ and $\psi, \varphi \in H(D)$ where $\varphi$ is a self-map of $D$. Then $\psi C_{\varphi}: H \Lambda_{\alpha} \rightarrow H \Lambda_{\beta}$ is bounded if and only if the following hold:

(1) $\psi \in H \Lambda_{\beta}$;

(2) $|\psi|(1-|\varphi|)^{\alpha} \in \Lambda_{\beta}(D)$.

In the next theorem we obtain a result corresponding to Theorem 2.6 for little analytic Lipschitz spaces.

THEOREM 2.7. Let $0<\alpha, \beta<1$ and $\psi, \varphi \in H(D)$ where $\varphi$ is a self-map of $D$. Then $\psi C_{\varphi}: H \Lambda_{\alpha}^{0} \rightarrow H \Lambda_{\beta}^{0}$ is bounded if and only if the following hold:

(1) $\psi \in H \Lambda_{\beta}^{0}$;

(2) $|\psi|(1-|\varphi|)^{\alpha} \in \Lambda_{\beta}(D)$;

(3) $\psi \varphi \in H \Lambda_{\beta}^{0}$.

PROOF. By [12, Theorem 4.1], $\psi C_{\varphi}: H \Lambda_{\alpha}^{0} \rightarrow H \Lambda_{\beta}^{0}$ is bounded if and only if the following hold:

(1)* $\psi \in H \Lambda_{\beta}^{0}$;

(2)* $\psi C_{\varphi}: H \Lambda_{\alpha} \rightarrow H \Lambda_{\beta}$ is bounded;

(3)* $\lim _{|z| \rightarrow 1}(1-|z|)^{1-\beta}|\psi(z)|\left|\varphi^{\prime}(z)\right|=0$.

Let $\psi C_{\varphi}$ be bounded; then by Theorem 2.6 it is enough to show that (3) holds. Clearly,

$$
\left|(\psi \varphi)^{\prime}(z)\right|(1-|z|)^{1-\beta} \leq\left|\psi^{\prime}(z)\right||\varphi(z)|(1-|z|)^{1-\beta}+|\psi(z)|\left|\varphi^{\prime}(z)\right|(1-|z|)^{1-\beta} .
$$

Since $\varphi$ is bounded and $\psi \in H \Lambda_{\beta}^{0}, \lim _{|z| \rightarrow 1}\left|\psi^{\prime}(z)\right||\varphi(z)|(1-|z|)^{1-\beta}=0$ by Lemma 2.1. Also $\lim _{|z| \rightarrow 1}|\psi(z)|\left|\varphi^{\prime}(z)\right|(1-|z|)^{1-\beta}=0$ by (3)* Therefore (2.6) 
implies that $\left|(\psi \varphi)^{\prime}(z)\right|(1-|z|)^{1-\beta} \rightarrow 0$ as $|z| \rightarrow 1$, and hence $\psi \varphi \in H \Lambda_{\beta}^{0}$ by Lemma 2.1.

Conversely, let (1), (2) and (3) hold. To prove that $\psi C_{\varphi}$ is bounded, we only need to show that $(3)^{*}$ holds. Now,

$$
\begin{aligned}
\left|(1-|z|)^{1-\beta} \psi(z) \varphi^{\prime}(z)\right| \leq & \left|(1-|z|)^{1-\beta} \psi(z) \varphi^{\prime}(z)+(1-|z|)^{1-\beta} \psi^{\prime}(z) \varphi(z)\right| \\
& \quad+\left|(1-|z|)^{1-\beta} \psi^{\prime}(z) \varphi(z)\right| \\
= & (1-|z|)^{1-\beta}\left|(\psi \varphi)^{\prime}(z)\right|+(1-|z|)^{1-\beta}\left|\psi^{\prime}(z)\right||\varphi(z)| .
\end{aligned}
$$

Using the boundedness of $\varphi$ and (1), we have $\lim _{|z| \rightarrow 1}(1-|z|)^{1-\beta}\left|\psi^{\prime}(z)\right||\varphi(z)|=0$ by Lemma 2.1. Also (3) implies that $\lim _{|z| \rightarrow 1}(1-|z|)^{1-\beta}\left|(\psi \varphi)^{\prime}(z)\right|=0$. Therefore (2.7) implies (3)*, which completes the proof.

Now Theorem 1.2 is an immediate consequence of Proposition 2.5 and Theorem 2.7. Also Theorem 1.3 follows from Proposition 2.5 and the next result.

THEOREM 2.8. Let $0<\alpha, \beta<1$ and $\psi, \varphi \in H(D)$ where $\varphi$ is a self-map of $D$. Then the following statements are equivalent:

(i) $\psi C_{\varphi}: H \Lambda_{\alpha}^{0} \rightarrow H \Lambda_{\beta}^{0}$ is compact;

(ii) $\lim _{|z| \rightarrow 1}|\psi(z)|\left((1-|z|)^{1-\beta} /(1-|\varphi(z)|)^{1-\alpha}\right)\left|\varphi^{\prime}(z)\right|=0$ and $\psi \in H \Lambda_{\beta}^{0}$;

(iii) $|\psi|(1-|\varphi|)^{\alpha} \in \Lambda_{\beta}^{0}(D)$ and $\psi \in H \Lambda_{\beta}^{0}$.

Proof. (i) $\Leftrightarrow$ (ii). This holds by [12, Theorem 5.1], where little Bloch type spaces are considered instead of little analytic Lipschitz spaces.

(iii) $\Rightarrow$ (ii). By (1.2), for each $z \in D$ there exists $w_{z} \in D$ such that $\left|w_{z}-z\right|<$ $1-|z|$ and

$$
\left|\varphi^{\prime}(z)\right| \leq 4 \frac{\left|\varphi\left(w_{z}\right)\right|-|\varphi(z)|}{1-|z|} .
$$

On the other hand, using (2.8) and the inequality $\alpha x^{\alpha-1}(x-y) \leq x^{\alpha}-y^{\alpha}$ for $0<$ $y<x$

$$
\begin{aligned}
\frac{\alpha}{4}\left|\varphi^{\prime}(z)\right| \frac{(1-|z|)^{1-\beta}}{(1-|\varphi(z)|)^{1-\alpha}} & \leq \frac{1}{4}\left|\varphi^{\prime}(z)\right|(1-|z|) \frac{\alpha(1-|\varphi(z)|)^{\alpha-1}}{\left|w_{z}-z\right|^{\beta}} \\
& \leq \frac{\alpha(1-|\varphi(z)|)^{\alpha-1}\left(\left|\varphi\left(w_{z}\right)\right|-|\varphi(z)|\right)}{\left|w_{z}-z\right|^{\beta}} \\
& \leq \frac{\left|(1-|\varphi(z)|)^{\alpha}-\left(1-\left|\varphi\left(w_{z}\right)\right|\right)^{\alpha}\right|}{\left|w_{z}-z\right|^{\beta}}
\end{aligned}
$$

Let $h(z):=|\psi(z)|(1-|\varphi(z)|)^{\alpha}$; then (2.9) gives

$$
\begin{aligned}
& \frac{\alpha}{4}|\psi(z)| \frac{(1-|z|)^{1-\beta}}{(1-|\varphi(z)|)^{1-\alpha}}\left|\varphi^{\prime}(z)\right| \\
& \quad \leq|\psi(z)| \frac{\left|(1-|\varphi(z)|)^{\alpha}-\left(1-\left|\varphi\left(w_{z}\right)\right|\right)^{\alpha}\right|}{\left|w_{z}-z\right|^{\beta}}
\end{aligned}
$$




$$
\begin{aligned}
\leq & \left|\frac{|\psi(z)|\left\{(1-|\varphi(z)|)^{\alpha}-\left(1-\left|\varphi\left(w_{z}\right)\right|\right)^{\alpha}\right\}-\left(1-\left|\varphi\left(w_{z}\right)\right|\right)^{\alpha}\left(\left|\psi\left(w_{z}\right)\right|-|\psi(z)|\right)}{\left|w_{z}-z\right|^{\beta}}\right| \\
& +\left(1-\left|\varphi\left(w_{z}\right)\right|\right)^{\alpha} \frac{|| \psi\left(w_{z}\right)|-| \psi(z)||}{\left|w_{z}-z\right|^{\beta}} \\
\leq & \frac{\left|h(z)-h\left(w_{z}\right)\right|}{\left|z-w_{z}\right|^{\beta}}+\left(1-\left|\varphi\left(w_{z}\right)\right|\right)^{\alpha} \frac{\left|\psi\left(w_{z}\right)-\psi(z)\right|}{\left|w_{z}-z\right|^{\beta}}
\end{aligned}
$$

Since $h, \psi \in \Lambda_{\beta}^{0}(D)$ and $\left|w_{z}-z\right|<1-|z|$, then

$$
\frac{\left|h(z)-h\left(w_{z}\right)\right|}{\left|z-w_{z}\right|^{\beta}} \rightarrow 0
$$

and

$$
\frac{\left|\psi\left(w_{z}\right)-\psi(z)\right|}{\left|w_{z}-z\right|^{\beta}} \rightarrow 0 \quad \text { as }|z| \rightarrow 1 .
$$

Therefore (2.11) implies that

$$
|\psi(z)| \frac{(1-|z|)^{1-\beta}}{(1-|\varphi(z)|)^{1-\alpha}}\left|\varphi^{\prime}(z)\right| \rightarrow 0 \quad \text { as }|z| \rightarrow 1 .
$$

(ii) $\Rightarrow$ (iii). First note that since $\psi$ and $\varphi$ are analytic, $|\nabla| \psi||=\left|\psi^{\prime}\right|$ and $\left|\nabla(1-|\varphi|)^{\alpha}\right|=\alpha\left|\varphi^{\prime}\right|(1-|\varphi|)^{\alpha-1}$. Therefore with the previous notation for $h(z)$ we get

$$
\begin{aligned}
|\nabla h(z)| & (1-|z|)^{1-\beta} \\
= & \left((1-|\varphi(z)|)^{\alpha}|\nabla| \psi(z)||+|\psi(z)|\left|\nabla(1-|\varphi(z)|)^{\alpha}\right|\right)(1-|z|)^{1-\beta} \\
= & (1-|\varphi(z)|)^{\alpha}\left|\psi^{\prime}(z)\right|(1-|z|)^{1-\beta} \\
& +\alpha|\psi(z)| \frac{(1-|z|)^{1-\beta}}{(1-|\varphi(z)|)^{1-\alpha}}\left|\varphi^{\prime}(z)\right| .
\end{aligned}
$$

Since $\psi \in H \Lambda_{\beta}^{0}$, by Lemma 2.1 we have $\lim _{|z| \rightarrow 1}\left|\psi^{\prime}(z)\right|(1-|z|)^{1-\beta}=0$. Thus, the hypothesis of part (ii), the boundedness of $\varphi$, and (2.11) imply that

$$
\lim _{|z| \rightarrow 1}\left|\nabla\left(|\psi(z)|(1-|\varphi(z)|)^{\alpha}\right)\right|(1-|z|)^{1-\beta}=0,
$$

and thus by Lemma 2.2, $|\psi|(1-|\varphi|)^{\alpha} \in \Lambda_{\beta}^{0}(D)$.

Let $0<\alpha \leq 1,0<\beta<1$ and $\varphi: D \rightarrow D$ belong to $H \Lambda_{\beta}^{0}$. Then by a similar argument as in [10, Proposition 2.2], one can prove that

$$
\lim _{|z| \rightarrow 1}|\psi(z)| \frac{(1-|z|)^{1-\beta}}{(1-|\varphi(z)|)^{1-\alpha}}\left|\varphi^{\prime}(z)\right|=0
$$

if and only if

$$
\lim _{|\varphi(z)| \rightarrow 1}|\psi(z)| \frac{(1-|z|)^{1-\beta}}{(1-|\varphi(z)|)^{1-\alpha}}\left|\varphi^{\prime}(z)\right|=0,
$$


where $\psi$ is an arbitrary bounded map on $D$. Using this fact, along with [12, Theorem 3.1], leads to the following result for analytic Lipschitz spaces and therefore, by Proposition 2.4, for Zygmund type spaces.

Corollary 2.9. Let $0<\alpha \leq 1,0<\beta<1$ and $\psi, \varphi \in H \Lambda_{\beta}^{0}$ where $\varphi$ is a selfmap of D. Then $\psi C_{\varphi}: H \Lambda_{\alpha} \rightarrow H \Lambda_{\beta}$ is compact if and only if $|\psi|(1-|\varphi|)^{\alpha} \in$ $\Lambda_{\beta}^{0}(D)$.

Note that if $g=\varphi^{\prime}$, then

$$
\left(C_{\varphi}^{\varphi^{\prime}} f\right)(z)=\left(C_{\varphi} f\right)(z)-\left(C_{\varphi} f\right)(0) .
$$

Therefore, $C_{\varphi}$ is bounded (compact) if and only if $C_{\varphi}^{\varphi^{\prime}}$ is bounded (compact). So one can apply Theorems 1.1, 1.2, and 1.3 to obtain necessary and sufficient conditions for boundedness and compactness of composition operators on Zygmund type spaces. In particular, we have the following derivative-free conditions for boundedness and compactness of composition operators on little Zygmund type spaces.

COROLlary 2.10. Let $0<\alpha, \beta<1$ and $\varphi$ be an analytic self-map of $D$. Then:

(i) $\quad C_{\varphi}: \mathcal{Z}_{0}^{\alpha} \rightarrow \mathcal{Z}_{0}^{\beta}$ is bounded if and only if $\varphi \in \mathcal{Z}_{0}^{\beta} \cap \mathcal{B}_{0}^{\beta / 2}$ and $(1-|\varphi|)^{1-\alpha / 2} \in$ $\Lambda_{1-\beta / 2}(D)$;

(ii) $C_{\varphi}: \mathcal{Z}_{0}^{\alpha} \rightarrow \mathcal{Z}_{0}^{\beta}$ is compact if and only if $\varphi \in \mathcal{Z}_{0}^{\beta}$ and $(1-|\varphi|)^{1-\alpha / 2} \in$ $\Lambda_{1-\beta / 2}^{0}(D)$.

PROof. To prove (ii), it is enough to use the equivalence of (i) and (ii) in Theorem 1.3 for $g=\varphi^{\prime}$ and then the equivalence of (ii) and (iii) for $g=1$. The proof of part (i) follows from a similar argument using Theorem 1.2, the equivalence of (B) and (C) in [14, Theorem A], Theorem 2.6, and

$$
(1-|z|)^{\beta}\left(\varphi^{\prime} \varphi\right)^{\prime}(z)=(1-|z|)^{\beta} \varphi^{\prime \prime}(z) \varphi(z)+\left((1-|z|)^{\beta / 2} \varphi^{\prime}(z)\right)^{2} .
$$

As a final remark, let us for $\alpha>0$ consider the standard weighted Banach spaces of analytic functions defined as follows:

$$
\begin{gathered}
H_{\alpha}^{\infty}=\left\{f \in H(D):\|f\|_{H_{\alpha}^{\infty}}=\sup _{z \in D}(1-|z|)^{\alpha}|f(z)|<\infty\right\}, \\
H_{\alpha}^{0}=\left\{f \in H_{\alpha}^{\infty}: \lim _{|z| \rightarrow 1}(1-|z|)^{\alpha}|f(z)|=0\right\} .
\end{gathered}
$$

For more details about spaces of this type we refer to $[1,2,7,11]$ and the references therein. Let $\gamma>0$ and consider the bounded operators

$$
\begin{gathered}
S: \mathcal{B}^{\gamma} \rightarrow H_{\gamma}^{\infty}, \quad S h=h^{\prime} ; \\
T: H_{\gamma}^{\infty} \rightarrow \mathcal{B}^{\gamma}, \quad(T h)(z)=\int_{0}^{z} h(\xi) d \xi .
\end{gathered}
$$


Then by the same arguments as in Propositions 2.4 and 2.5, one can reduce the study of generalized composition operators $C_{\varphi}^{g}: \mathcal{B}^{\alpha} \rightarrow \mathcal{B}^{\beta}$ and $C_{\varphi}^{g}: \mathcal{B}_{0}^{\alpha} \rightarrow \mathcal{B}_{0}^{\beta}$ to weighted composition operators $g C_{\varphi}: H_{\alpha}^{\infty} \rightarrow H_{\beta}^{\infty}$ and $g C_{\varphi}: H_{\alpha}^{0} \rightarrow H_{\beta}^{0}$. Indeed, the following diagram commutes:

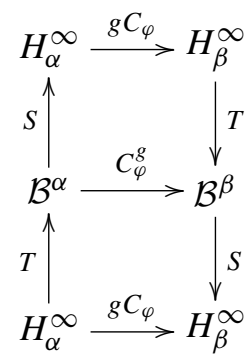

Therefore, the following proposition holds.

Proposition 2.11. Let $\alpha, \beta>0$ and $g, \varphi \in H(D)$ where $\varphi$ is a self-map of $D$. Then:

(i) $C_{\varphi}^{g}: \mathcal{B}^{\alpha} \rightarrow \mathcal{B}^{\beta}$ is bounded (compact) if and only if $g C_{\varphi}: H_{\alpha}^{\infty} \rightarrow H_{\beta}^{\infty}$ is bounded (compact);

(ii) $C_{\varphi}^{g}: \mathcal{B}_{0}^{\alpha} \rightarrow \mathcal{B}_{0}^{\beta}$ is bounded (compact) if and only if $g C_{\varphi}: H_{\alpha}^{0} \rightarrow H_{\beta}^{0}$ is bounded (compact).

As a result of Proposition 2.11, one can easily obtain several of the results in [6, Section 5] by applying [11, Theorems 2.1 and 2.2].

\section{Acknowledgement}

The research for this paper was carried out while the second author was visiting University of Oulu, whose hospitality is acknowledged with thanks.

\section{References}

[1] K. D. Bierstedt, J. Bonet and J. Taskinen, 'Associated weights and spaces of holomorphic functions', Studia Math. 127(2) (1998), 137-168.

[2] J. Bonet, P. Domański, M. Lindström and J. Taskinen, 'Composition operators between weighted Banach spaces of analytic functions', J. Aust. Math. Soc. Ser. A 64(1) (1998), 101-118.

[3] B. R. Choe, H. Koo and W. Smith, 'Composition operators on small spaces', Integral Equations Operator Theory 56(3) (2006), 357-380.

[4] P. L. Duren, Theory of $H^{p}$ Spaces, Pure and Applied Mathematics, 38 (Academic Press, San Diego, CA, 1970).

[5] K. M. Dyakonov, 'Equivalent norms on Lipschitz-type spaces of holomorphic functions', Acta Math. 178(2) (1997), 143-167.

[6] S. Li and S. Stević, 'Generalized composition operators on Zygmund spaces and Bloch type spaces', J. Math. Anal. Appl. 338(2) (2008), 1282-1295.

[7] W. Lusky, 'On weighted spaces of harmonic and holomorphic functions', J. Lond. Math. Soc. 51 (1995), 309-320.

[8] K. M. Madigan, 'Composition operators on analytic Lipschitz spaces', Proc. Amer. Math. Soc. 119(2) (1993), 465-473. 
[9] K. M. Madigan and A. Matheson, 'Compact composition operators on the Bloch space', Trans. Amer. Math. Soc. 347(7) (1995), 2679-2687.

[10] A. Montes-Rodríguez, 'The essential norm of a composition operator on Bloch spaces', Pacific J. Math. 188(2) (1999), 339-351.

[11] A. Montes-Rodríguez, 'Weighted composition operators on weighted Banach spaces of analytic functions', J. Lond. Math. Soc. (2) 61(3) (2000), 872-884.

[12] S. Ohno, K. Stroethoff and R. Zhao, 'Weighted composition operators between Bloch-type spaces', Rocky Mountain J. Math. 33(1) (2003), 191-215.

[13] M. Pavlović, 'On Dyakonov's paper: equivalent norms on Lipschitz-type spaces of holomorphic functions', Acta Math. 183(1) (1999), 141-143.

[14] M. Pavlović, 'Derivative-free characterizations of bounded composition operators between Lipschitz spaces', Math. Z. 258(1) (2008), 81-86.

[15] R. C. Roan, 'Composition operators on a space of Lipschitz functions', Rocky Mountain J. Math. 10(2) (1980), 371-379.

[16] W. Rudin, Function Theory in the Unit Ball of $\mathbb{C}^{n}$, Grundlehren der Mathematischen Wissenschaften, 241 (Springer, New York, 1980).

[17] K. Zhu, Spaces of Holomorphic Functions in the Unit Ball, Graduate Texts in Mathematics, 226 (Springer, New York, 2005).

MIKAEL LINDSTRÖM, Department of Mathematical Sciences, University of Oulu, 90014 Oulu, Finland

e-mail: mikael.lindstrom@oulu.fi

AMIR H. SANATPOUR, Department of Mathematics, Tarbiat Moallem University, 599 Taleghani Avenue, 15618 Tehran, Iran

e-mail: a_sanatpour@tmu.ac.ir 